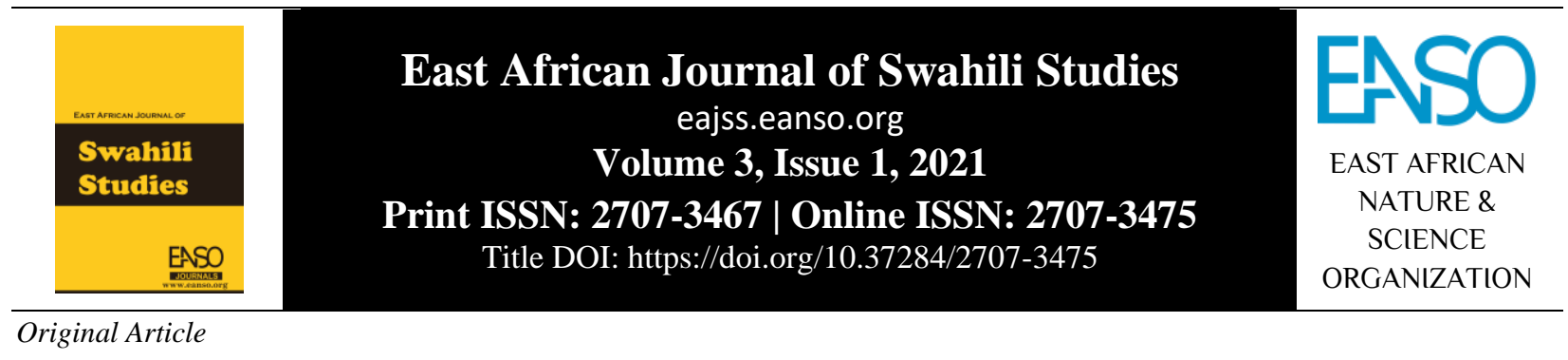

\title{
Tathmini Linganishi kati ya Mtalaa wa 8.4.4 na wa Umilisi wa 2.6.6.3 katika Ufundishaji wa Fonimu za Kiswahili katika Kiwango cha Chekechea
}

\author{
Nabangi Joan Wataka ${ }^{*}$, Susan C. Choge, PhD ${ }^{1} \&$ Luganda Musavi Manasseh ${ }^{1}$ \\ ${ }^{1}$ Idara ya Lugha na Fasihi, Chuo Kikuu cha Sayansi na Teknolojia cha Masinde Muliro. \\ * ORCID: https://orcid.org/0000-0003-2319-6088; Barua pepe ya mawasiliano: watakajoan@gmail.com.
}

DOI ya Nakala: https://doi.org/10.37284/eajss.3.1.267

\section{Tarehe ya Uchapishaji: IKISIRI}

08 Januari 2021 Mtalaa ni mpangilio ambao huweka malengo katika ufunzaji wa wanafunzi. Unatumika kama mwongozo kwa walimu ambao huweka viwango vya Istilahi Muhimu: matokeo ya mwanafunzi sawia na uwajibikaji wa mwalimu. Nchi ya Kenya imeshuhudia mabadiliko ya mitalaa mbalimbali tangu ilipojinyakulia uhuru. Mtalaa, Nafasi ya mtalaa wa uhuru wa 7.4.2.3 ilichukuliwa na 8.4.4 mwaka wa 1985 Mtalaa wa Umilisi, ambao nafasi yake kwa sasa inachukuliwa na mtalaa mpya wa umilisi wa Nadharia ya Utambuzi, Fonimu za Kiswahili, Chekechea. 2.6.6.3 ambao ulianzishwa mwaka wa 2017. Mitalaa hii imefafanua vipengele mbalimbali kwa kutumia mbinu mbalimbali za ufundishaji na ufunzaji. Kwa hivyo, lengo la nakala hii ni kutathmini kiulinganishi ufundishaji wa fonimu za Kiswahili katika kiwango cha chekechea katika mtalaa wa 8.4.4 unaoondolewa, na katika mtalaa mpya wa umilisi wa 2.6.6.3. Mtalaa wa 7.4.2.3 na wa 8.4.4 iliegemea sana uwezo wa mwalimu, yeye ndiye aliyechukua jukumu kubwa katika mchakato wa ufundishaji kuliko wanafunzi. Tofauti na mitalaa hiyo miwili, mtalaa wa 2.6.6.3 unampa mwanafunzi jukumu kubwa katika mchakato wa ujifunzaji kuliko mwalimu. Makala haya pia yamechunguza kiulinganishi ubora na udhaifu wa mbinu mbalimbali za ufundishaji wa fonimu za Kiswahili katika kiwango cha chekechea katika mitalaa ya 8.4.4 na mpya wa 2.6.6.3. Nadharia ya Piaget ya Utambuzi imeongoza makala haya. Makala haya yanaripoti baadhi ya matokeo ya utafiti uliofanywa kwa minajili ya shahada ya uzamili katika Elimu ya Kiswahili.

\section{APA CITATION}

Wataka, N. J., Choge, S. C., \& Manasseh, L. (2021). Tathmini Linganishi kati ya Mtalaa wa 8.4.4 na wa Umilisi wa 2.6.6.3 katika Ufundishaji wa Fonimu za Kiswahili katika Kiwango cha Chekechea. East African Journal of Swahili Studies, 3(1), 1-9. https://doi.org/10.37284/eajss.3.1.267.

1 | This work is licensed under a Creative Commons Attribution 4.0 International License. 


\section{CHICAGO CITATION}

Wataka, Nabangi Joan, Susan C. Choge, and Luganda Musavi Manasseh. 2021. "Tathmini Linganishi kati ya Mtalaa wa 8.4.4 na wa Umilisi wa 2.6.6.3 katika Ufundishaji wa Fonimu za Kiswahili katika Kiwango cha Chekechea". East African Journal of Swahili Studies 3 (1), 1-9. https://doi.org/10.37284/eajss.3.1.267.

\section{HARVARD CITATION}

Wataka, N. J., Choge, S. C. and Manasseh, L. (2021) "Tathmini Linganishi kati ya Mtalaa wa 8.4.4 na wa Umilisi wa 2.6.6.3 katika Ufundishaji wa Fonimu za Kiswahili katika Kiwango cha Chekechea", East African Journal of Swahili Studies, 3(1), pp. 1-9. doi: 10.37284/eajss.3.1.267.

\section{IEEE CITATION}

N. J. Wataka, S. C. Choge, and L. Manasseh, "Tathmini Linganishi kati ya Mtalaa wa 8.4.4 na wa Umilisi wa 2.6.6.3 katika Ufundishaji wa Fonimu za Kiswahili katika Kiwango cha Chekechea”, EAJSS, vol. 3, no. 1, pp. 1-9, Jan. 2021.

\section{MLA CITATION}

Wataka, Nabangi Joan, Susan C. Choge, and Luganda Musavi Manasseh. "Tathmini Linganishi kati ya Mtalaa wa 8.4.4 na wa Umilisi wa 2.6.6.3 katika Ufundishaji wa Fonimu za Kiswahili katika Kiwango cha Chekechea”. East African Journal of Swahili Studies, Vol. 3, no. 1, Jan. 2021, pp. 1-9, doi:10.37284/eajss.3.1.267.

\section{UTANGULIZI}

Makala haya yanatathmini kiulinganishi mtalaa wa 8.4.4 na wa 2.6.6.3 unaozingatia umilisi katika ufundishaji wa fonimu za Kiswahili katika shule za chekechea. Katika kuchunguza suala hili, makala yameshughulikia vipengele kama vile, maelezo kuhusu maana ya mtalaa na sifa zake kwa kuzingatia maoni ya wataalamu anuwai. Vilevile, makala haya yametoa ufafanuzi kuhusu mfumo wa fonimu za Kiswahili kwa jumla kwa nia ya kuimarisha uelewa wa msomaji kuhusu suala linaloshughulikiwa na makala.

Nadharia ya Utambuzi iliyoasisiwa na Piaget (1972) imeongoza uandishi wa makala haya. Nadharia hii imefafanuliwa na kuhusishwa na makala kwa kuonyesha jinsi ilivyosaidia katika kuikuza. Kwa vile makala haya yametalii mitalaa miwili tofauti kiulinganishi, kuna ufafanuzi wa kijumla kuhusu mitalaa hiyo kwa kurejelea mbinu zinazotumiwa na kila mtalaa kufundishia fonimu za Kiswahili katika kiwango cha chekechea. Mbinu hizi zimelinganishwa kwa kuhusisha ufaafu na udhaifu na namna ufaafu au udhaifu huo huchangia ufanisi wa ufundishaji fonimu za Kiswahili. Matokeo ya mbinu za ufundishaji kwa mujibu wa kila mtalaa yamefafanuliwa kiulinganishi ili kutimiza maazimio ya mada ya makala haya. Mapendekezo ya jinsi ya kuimarisha na kuboresha ufundishaji pia yametolewa.

\section{Mtalaa na sifa zake}

Mtalaa ni mfumo ambao hutoa maelekezo kuhusu mawanda ya masomo mbalimbali katika kiwango fulani cha elimu na jinsi ambavyo masomo hayo yanafaa kufunzwa, mbinu za ufundishaji na matokeo yanayotarajiwa.

Flinders na Thorntorn (2004) wanafafanua mtalaa kwa njia mbili. Kwanza, wanasema kuwa mtalaa ni tajriba zote zinazodhamiriwa kuchochea uwezo wa mtu. Pili, wanasema kuwa, mtalaa ni msururu wa tajriba ambazo zinalenga kufunzwa zinazotumiwa na taasisi za elimu kuafikia mchakato wa kuibua uwezo wa mwanafunzi. Kwao, mtalaa una majukumu muhimu katika mfumo wa elimu; ya kuchochea uwezo wa kiung'amuzi wa mwanafunzi na tena kuainisha utaratibu wa tajriba ambazo zinadhamiriwa kufunzwa katika mazingira ya elimu.

Kridel (2010) wanasema kwamba, mtalaa ni jumla ya tajriba ambazo zinapatikana na kupangika kwa jinsi ya kusababisha ujifunzaji. Kulingana na maelezo haya, ni kweli kwamba, mtalaa huhusishwa na shughuli za ufundishaji. Pia, mtalaa sharti uwe na mpangilio mahususi kwa sababu hudhamiriwa kutoa maelekezo katika mchakato wa ufundishaji na ujifunzaji.

Chiduo na wengine (2016), wanaeleza kuwa, mtalaa ni mpango wa masomo ya kiwango mahususi cha elimu. Kutokana na ufafanuzi wao, inabainika kuwa, viwango mbalimbali vya kielimu

2 | This work is licensed under a Creative Commons Attribution 4.0 International License. 
vina mitalaa yao. Hii ni thibitisho kwamba, mtalaa una umuhimu katika kufanikisha elimu endelevu.

Kwa hivyo, mtalaa una majukumu muhimu katika mchakato wa ufundishaji na ujifunzaji wa masomo mbalimbali katika viwango tofauti vya elimu. Dhamira kuu ya mtalaa ni kutoa utaratibu na vigezo ambavyo hutumiwa na walimu na wanafunzi kufanikisha elimu.

Ili kutimiza malengo ya elimu, ni sharti mtalaa uwe na sifa maalum ambazo zitauwezesha kutoa mwongozo uliokusudiwa kwa njia mwafaka. Sifa hizi za mtalaa zinaweza zikawa za kimuundo ama za kimaelezo ili kufanikisha elimu.

Wandera (2018) anaposhughulikia suala la sifa za mtalaa bora, anasema kwamba, mtalaa bora sharti ushughulikie mahitaji ya wanafunzi kwa namna inayofaa. Anaeleza kuwa, wanafunzi mbalimbali huwa na tofauti za kibinafsi, mtalaa bora lazima uzingatie tofauti hizi na kuzishughulikia kwa usawa. Katika kukuza mitalaa, tofauti hizi za kiuwezo miongoni mwa wanafunzi ni muhimu zikizingatiwa. Hii ndiyo sababu ya kuhusishwa kwa masomo ya lazima, ya hiari na masomo ya ziada katika mtalaa. Hii huwapa wanafunzi fursa ya kujichagulia masomo yao kutegemea mahitaji yao yanayotokana na upekee wao.

Aidha, Wandera (2018) anaeleza kuwa, ni jukumu la wizara ya elimu kuwahusisha washikadau katika sekta ya elimu wakati wa kuunda na kurekebisha mitalaa. Katika kufanya haya, mahitaji ya kisaikolojia ya wanafunzi lazima yazingatiwe kikamilifu. Anahimiza kwamba, mtalaa bora sharti uzingatie upeo wa mwanafunzi. Mada za mtalaa vilevile sharti ziambatane na mahitaji ya kisaikolojia, mapendeleo na tabia za kiasilia za wanafunzi. Kwa jinsi hiyo, ni lazima mtalaa uzingatie uwezo mbalimbali wa wanafunzi kwa kiwango sawa. Kwa hivyo, mtalaa unafaa kukuzwa ili uambatane na mahitaji ya mwanafunzi bali si mwanafunzi kujilazimisha kufuata mahitaji ya mtalaa. Hii ni kwa sababu mtalaa unapokuzwa hadi kiwango kinachomshinda mwanafunzi kufikia, basi mwanafunzi atakosa hamu na ari ya kuendelea na elimu yake. Mtalaa unapofeli kumzingatia mwanafunzi basi unapoteza dira na kusalia bila maana yoyote katika elimu ya mwanafunzi.

\section{Mfumo wa fonimu za Kiswahili kwa jumla}

Mgullu (2016) akimnukuu Trubetzkoy (1939) anasema kuwa, fonimu ni kipashio kidogo kabisa katika lugha kilicho na uamilifu wa kutofautisha maana katika maneno. Maelezo ya Trubetzkoy yanaonyesha majukumu na umuhimu wa fonimu katika lugha.

Kwa mujibu wa Kamusi ya Karne ya 21, Toleo la 4 (2019), fonimu ni kipandesauti katika neno ambacho kikibadilishwa na kipandesauti kingine maana ya neno hilo hubadilika katika lugha hiyo. Maelezo haya yanaoana na maelezo ya Trubetzkoy kwa kuwa, iwapo fonimu itabadilishwa katika neno na nafasi yake kuchukuliwa na fonimu nyingine, basi neno hilo hubadilisha maana pia.

Makala haya yanashughulikia aina mbili za fonimu za Kiswahili ambazo ni; fonimu za irabu na fonimu za konsonanti.

\section{Mfumo wa Irabu katika Kiswahili}

Mfumo wa irabu katika Kiswahili umetafitiwa na wataalamu mbalimbali kama vile, Massamba na wenzake (2004), Mgullu (2016) na Matinde (2012). Wataalamu hawa wameafikiana kuwa Kiswahili kina jumla ya fonimu za irabu tano ambazo ni /a//e/ /i/ /o/ /u/.

\section{Mfumo wa Konsonanti katika Kiswahili}

Wanaisimu mbalimbali wamefanya uchunguzi kuhusu konsonanti katika Kiswahili. Hata hivyo, wanaisimu hawa hawajakubaliana kuhusu idadi kamili ya konsonanti za Kiswahili. Baadhi ya wanaisimu kama vile, Mgullu (2016) alianisha konsonanti ishirini na nne, Massamba na wenzake (2004) walianisha konsonanti ishirini na tisa.

Massamba na wenzake (2004) katika uanishaji wao, wakaeleza kuwa, sifa muhimu ya kubainisha fonimu ni mpumuo kwa kuwa ni sifa bainifu katika Kiswahili sanifu. Kwa hivyo, waliorodhesha sauti zifuatazo zilizo na mpumuo ( $\mathrm{ph}$, th, ch, kh). Hata hivyo, makala haya yanaunga mkono mawazo ya Mgullu (2016) kuwa, suala la kuwepo au kutokuwepo kwa mpumuo katika baadhi ya konsonanti za Kiswahili sanifu halifai kuingizwa kwa sababu katika Kiswahili sanifu mpumuo hautumiwi kama kigezo cha kutofautisha maana za

3 | This work is licensed under a Creative Commons Attribution 4.0 International License. 
maneno. Ikiwa mpumuo ungekuwa unatumiwa kutofautisha maana, bila shaka konsonanti yenye mpumuo ingekuwa ni fonimu tofauti na ile isiyo na mpumuo.

Kuna watafiti wengi ambao wametafitia fonimu za irabu na fonimu za konsonanti za Kiswahili.

Okal (2017) katika utafiti wake kuhusu uhakiki wa fonimu na miundo ya silabi za Kiswahili alieleza kuwa, utambuzi wa fonimu za Kiswahili ungali unaendelea na fonimu zenyewe hufaa kurekodiwa mara kwa mara zinapotambuliwa (Choge, 2009). Iwapo hakuna alama katika orodha ya alama za unukuzi makinifu ya kimataifa basi alama mpya au alama saidizi huweza kubuniwa na wanaisimu wa lugha husika (Lyons, 1981). Alama saidizi hutumika katika lugha nyingine za Afrika, Marekani Kusini, Asia Kusini na Pasifika kuwakilisha fungu fonimu zilizokubalika kama vile, fonimu $/ \mathrm{mb}, \overline{n d} /$ zinazotamkwa kama sauti moja (Anderson, 1976 akirejelewa na Mwita, 2007).

Okal anaendelea kwa kusema kuwa, utambuzi wa fonimu za lugha hauna kikomo. Hii ndiyo sababu Choge (2009) anasema kuwa, utambuzi na uwekaji rekodi wa fonimu za Kiswahili ungali unaendelea. Utambuzi huo wa fonimu za Kiswahili huonekana katika sauti kama vile /N, D, <b, N/ (Nchimbi, 1992 akirejelewa katika Choge, 2009). Katika kuonyesha kuwa, utambuzi wa fonimu ungali unaendelea, Choge (2009) anatambua fonimu irabu nyingine za Kiswahili kama vile, irabu ndefu /i:, $\varepsilon:$ a:, u: \& כ:/ na irabu unganifu /au \& כa/ na kuzidhihirisha katika data maalumu. Choge (2009) alieleza majukumu ya fonimu za irabu au vokali za Kiswahili. Hata hivyo, sehemu muhimu ya utafiti wake inachangia katika kuelewa idadi kamili ya fonimu za Kiswahili. Alieleza kuwa, utambuzi na uandishi kuhusu fonimu za Kiswahili ni mchakato ambao ungali unaendelea. Aliendelea kwa kueleza kuwa, wanaisimu wa awali walibaini kuwa, Kiswahili kilikuwa na fonimu ama 32 au 37. Kati ya fonimu hizi, walibaini irabu tano ambazo ni /a/, /e/, /i/, /o/ na /u/ pamoja na nusu irabu mbili ambazo ni /w/ na /y/. Utafiti huu unachangia katika kuelewa mtazamo wa wanaisimu kuhusu fonimu za Kiswahili ambazo ni sehemu muhimu ya makala haya. Kumekuwepo na mjadala kuhusu idadi kamili ya fonimu za Kiswahili na maandishi mengi yamenukuu idadi zinazotofautiana. Kwa hivyo, utafiti wa Choge ulitoa mwanga wa kuelewa mwelekeo huu wa mjadala kwa kuelewa kuwa ni suala la utafiti na uchunguzi unaendelea.

Okal anahitimisha kwa kueleza kwamba, 'sauti mwambatano huweza kufasiriwa kama fonimu moja iwapo imetamkwa katika sehemu moja ya utamkaji au kutokana na urahisishaji wa utamkaji changamano'. Maelezo haya ya Okal yanatoa uelewa wa mwelekeo kuhusu suala la fonimu za Kiswahili ambalo limezua mjadala kwa muda mrefu na linaendelea kuzua mijadala mingine mingi.

Powers (2008) anaeleza namna nzuri na mwafaka ya kuwasaidia wanafunzi wa viwango vya chini kuimarisha uelewa wao kuhusu fonimu ni usomaji wakiwa katika vikundi vidogo vidogo. Kupitia usomaji katika vikundi, mwalimu ataweza kubaini jinsi wanafunzi tofauti wanavyoelewa fonimu kwa viwango tofauti. Powers anaendelea kwa kueleza kuwa, iwapo wanafunzi wanaweza kuwa na uelewa wa fonimu mapema, basi usomaji wao utaimarika na kuboresha masomo yao. Maelezo ya utafiti huu wa Powers unatoa msingi wa kuelewa ni kwa nini wizara ya elimu kupitia kwa Taasisi ya Ukuzaji Mitalaa Nchini, (KICD) iliweka somo la fonimu lifunzwe kuanzia viwango vya chini vya elimu.

Facun-Granadozo (2014) alibaini kuwa, uelewa wa fonimu unawasaidia wanafunzi kuelewa masomo ya lugha wanayofundishwa vyema na kujielewa wenyewe. Pia, uelewa wa fonimu huwawezesha wanafunzi kutatua matatizo na changamoto wanazokumbana nazo katika mchakato wao wa kukata kiu ya masomo. Pia, matokeo ya utafiti wa Facun-Granadozo yalibaini kuwa, walimu, watekelezaji kanuni, wasimamizi wa shule pamoja na wadau wengine katika sekta ya elimu wanapaswa kulitilia maanani suala la fonimu wanapoandaa mitalaa ya kufundishia. Utafiti wa Facun-Granadozo unatoa mwanga wa kuelewa umuhimu wa ufundishaji wa fonimu kwa wanafunzi wa kiwango cha chekechea. Vilevile, unatoa maelezo ya kuwasaidia walimu kama waelekezi katika mchakato mzima wa ufundishaji wa fonimu kwa wanafunzi wa kiwango cha chekechea.

4 | This work is licensed under a Creative Commons Attribution 4.0 International License. 


\section{MISINGI YA KINADHARIA}

Nadharia ya Utambuzi iliyoasisiwa na Piaget (1972) inaangazia asili ya ufahamu na jinsi mwanadamu anavyopata ufahamu, anavyoumiliki na hatimaye anavyoutumia. Kilichomchochea Piaget kuasisi nadharia hii ni hali kwamba, watoto wa umri tofauti walikuwa wakifanya makosa tofauti wanapojaribu kujibu maswali wanayoulizwa. Pia, aliamini kwamba, watoto si 'binadamu wachanga' ambao wamepungukiwa na ufahamu, bali wana ufahamu wa kutosha ila tu wao huzungumza kwa njia tofauti na watu wazima. Kwa hivyo, Piaget alijiaminisha kwamba watoto nao wana uwezo mkubwa wa kiutambuzi. Piaget baada ya kutambua kwamba watoto wana uwezo mkubwa wa kiutambuzi, aliwagawa katika makundi mbalimbali kutegemea umri wao na kuanza kuchunguza ukuaji wa utambuzi miongoni mwa watoto wa umri mbalimbali. Baada ya uchunguzi huu, Piaget aligundua kwamba, watoto hujifunza kupitia kwa matendo, kuita vitu kwa majina na kwa kufikiri kiuchanganuzi. Kulingana na Piaget, ukuaji wa kiutambuzi ni mchakato unaohusisha kupanguliwa upya kwa ufahamu ambao husababisha mabadiliko ya kibayolojia na tajriba ya kiakili.

Bruner (1974), aliendeleza nadharia ya Utambuzi alipochunguza uwezo wa kiutambuzi wa watoto. Lakini Bruner alitofautiana na Piaget aliyetilia mkazo uwezo wa kindani wa mtoto pekee. Bruner alisisitiza majukumu ya mazingira na tajriba katika ukuaji wa mtoto kiakili. Yeye alichunguza maendeleo ya utambuzi katika lugha ya watoto kwa vile, alikuwa na ari ya kuchunguza lugha na uwasilishaji wa mawazo ya binadamu. Mintarafu ya uchunguzi aliofanya, Bruner alipendekeza kuwa, mwanafunzi anafaa kushiriki kikamilifu katika mchakato mzima wa kielimu. Hii ni kwa sababu ujifunzaji ni shughuli inayohusisha ubunifu ambao unashirikisha kuibuliwa kwa dhana mpya ambazo hujalizia zile ambazo tayari mtoto anazifahamu. Katika kutamatisha, Bruner anasema kwamba, kwa jumla, ujifunzaji hutokana na uwezo wa mwanafunzi kuzielewa hali na miktadha mipya anayokumbana nayo kila siku.

Kwa ujumla, nadharia ya Utambuzi imedokeza viambajengo mbalimbali ambavyo vinaweza kurejelewa katika ufundishaji. Viambajengo hivyo ni: nafasi ya mwalimu kama mwelekezi, uwezo tofauti wa utambuzi, tajriba za wanafunzi na nafasi ya ushiriki wa wanafunzi katika ujifunzaji.

Nadharia ya Utambuzi ilitumika kwa sababu ufundishaji wa fonimu kwa kuzingatia mtalaa wa 8.4.4 na wa 2.6.6.3 unalenga kuwawezesha wanafunzi kutambua aina tofauti za fonimu za lugha mahususi na jinsi ya kuzitamka fonimu hizo ipasavyo. Mtalaa wa umilisi wa 2.6.6.3 unashabihiana na vipengele vya nadharia ya Utambuzi ambavyo vinapendekeza kuwa uwezo wa kiutambuzi wa mwanafunzi ni kiungo muhimu sana katika ujifunzaji wake.

\section{MTALAA WA 8.4.4 NA WA 2.6.6.3 WA UMILISI KIULINGANISHI}

Sehemu hii imechunguza mtalaa wa 8.4.4 na wa umilisi wa 2.6.6.3, inafafanuliwa kiuliganishi kwa kuzingatia miongozo yao kuhusu ufundishaji wa fonimu za Kiswahili katika kiwango cha chekechea.

\section{Muhtasari wa Mtalaa wa 8.4.4}

Mtalaa wa 8.4.4 ulianzishwa nchini Kenya mnamo mwaka wa 1985 kuchukua pahali pa mtalaa wa 7.4.2.3, uliotumika tangu Kenya kujipatia uhuru. Katika mtalaa wa 8.4.4, wanafunzi walichukua muda wa miaka minane katika shule ya msingi, miaka minne katika shule ya sekondari na angalau miaka minne katika viwango vya juu vya masomo kama vile vyuo vikuu. Kiswahili kilifunzwa kama somo la lazima katika kiwango cha chekechea, shule ya msingi na shule ya upili. Wizara ya elimu ilitoa mwelekeo wa jinsi ya kufunza mada mbalimbali za Kiswahili kwenye silabasi. Katika mtalaa wa 8.4.4, Kiswahili kilitumiwa kama lugha ya kufundishia katika viwango vya chekechea.

\section{Muhtasari wa Mtalaa wa Umilisi wa 2.6.6.3}

Mwaka wa 2017, serikali ya rais Uhuru Kenyatta ikishirikiana na Taasisi ya Ukuzaji wa Mitalaa Nchini, (KICD) na washika dau kutoka nyanja zote ilipendekeza mtalaa wa umilisi wa 2.6.6.3. Hii ikiwa mara ya tatu nchi ya Kenya ikibadilisha mifumo yake ya elimu baada ya mfumo wa pili wa 8.4.4 kutumika kwa takriban miaka thelathini na miwili. Mtalaa wa 2.6.6.3 ulikuwa na malengo ya

5 | This work is licensed under a Creative Commons Attribution 4.0 International License. 
kuhakikisha kuwa wanafunzi wanahitimu wakiwa wajuzi, wafanisi na watendaji kinyume na ilivyokuwa katika mtalaa wa 8.4.4 ambapo wengi waling'ang'ania kupata alama za juu kwenye mitihani lakini wakakosa ujuzi wa taaluma walizosomea.

Katika mtalaa wa 2.6.6.3, mwanafunzi husoma miaka miwili katika chekechea, miaka sita katika shule ya msingi, miaka sita katika shule ya upili, kisha miaka mitatu katika viwango vya juu vya elimu kama vile chuo kikuu.

KICD (2019) inasema kwamba, mtalaa wa 2.6.6.3 umependekeza mwalimu kufuata mtindo wa ninasoma-tunasoma-unasoma (mwalimu asome, asome pamoja na wanafunzi kisha mwanafunzi asome) unaofanikisha umilisi na utendaji kinyume na ulivyo mtindo wa mtalaa wa 8.4.4. ambao wanafunzi waliiga aliyofunza mwalimu.

Kwa mujibu wa KICD (2019), mtalaa wa umilisi umejumuisha masuala tofauti na ambayo ni muhimu kwa kila mwanafunzi. Miongoni mwa masuala haya ni pamoja na umilisi wa kimsingi. Mtalaa huu umetambua umilisi tofauti ambao unapaswa kujengwa mwanafunzi anapoendeleza masomo yake. Umilisi huu ni pamoja na: mawasiliano na ushirikiano, uwazaji kina na utatuzi wa matatizo, ubunifu, uraia, ujuzi wa kidijitali hamu ya kuendelea kujifunza na kujiamini.

KICD inaeleza kuwa, inaaminika kwamba, mwanafunzi akipata umilisi ufaao, ataweza kufaa katika jamii inayobadilika kila uchao. Kutokana na imani hii, kazi katika mtalaa huu imepunguzwa ili mwalimu apate nafasi mwafaka kumsaidia mwanafunzi kujenga umilisi huu.

\section{MBINU ZA UFUNDISHAJI WA FONIMU ZA KISWAHILI KATIKA KIWANGO CHA CHEKECHEA KATIKA MTALAA WA 8.4.4 NA WA UMILISI WA 2.6.6.3 KIULINGANISHI}

Sehemu hii inaeleza mfanano na tofauti za mbinu mbalimbali za ufundishaji wa fonimu za Kiswahili kwa mujibu wa mtalaa wa 8.4.4 na wa umilisi wa 2.6.6.3.

\section{Mfanano wa mbinu za Ufundishaji wa Fonimu za Kiswahili katika Mtalaa wa 8.4.4 na wa Umilisi wa 2.6.6.3}

Mtalaa wa 8.4.4 na wa umilisi wa 2.6.6.3 ilipendekeza mbinu mbalimbali za kutumiwa kufanikisha ufundishaji wa fonimu za Kiswahili katika kiwango cha chekechea. Baadhi ya mbinu za ufundishaji ambazo zilipendekezwa na mitalaa ya hii ni:

(a) Chati - Hivi ni vipande vikubwa vya karatasi vyenye maandishi na michoro ambavyo aghalabu huangikwa ukutani na wanafunzi kushirikishwa katika usomaji wa maandishi yaliyomo. Chati hupendekezwa kama mbinu moja ya ufundishaji wa fonimu. Fonimu za Kiswahili huandikwa kwenye chati hizi kisha wanafunzi wakielekezwa na mwalimu huzisoma na kuzielewa fonimu hizo.

(b) Picha - Picha ni sura ya kitu au mtu ambayo aghalabu hunaswa kwa kamera ama kuchorwa na kuchapwa kwenye karatasi. Wanafunzi husoma majina ya picha na michoro huku matamshi ya majina hayo yakisisitizwa kwa nia ya kuwafunza fonimu za Kiswahili.

(c) Kadi - Hivi ni vipande vidogo vya karatasi maalum. Fonimu za Kiswahili huandikwa kwenye kadi hizi kisha mwalimu huziinua moja baada ya nyingine na kuwauliza wanafunzi wazisome fonimu wanazoziona kwenye kadi hizo. Mwalimu huwarekebisha wale ambao hawajazitamka ipasavyo.

(d) Vikundi - Hii ni mikusanyiko ya idadi maalum ya wanafunzi ambao hushiriki katika shughuli maalum darasani kwa mwongozo wa mwalimu. Mwalimu huwaweka wanafunzi pamoja kulingana na idadi, jinsia na uwezo wao darasani kisha kuwaelekeza kufanya mazoezi ama shughuli zozote zinazochangia ufundishaji wa fonimu za Kiswahili.

6 | This work is licensed under a Creative Commons Attribution 4.0 International License. 
Tofauti kati ya mbinu za Ufundishaji wa Fonimu za Kiswahili katika Mtalaa wa 8.4.4 na wa Umilisi wa 2.6.6.3

Kuwepo kwa mtalaa wa umilisi wa 2.6.6.3 kumeleta mbinu mpya za ufundishaji wa fonimu za Kiswahili ambazo hazikutumika katika mtalaa wa 8.4.4. Baadhi ya mbinu hizo ni kama zifuatazo:

(a) Kanda za kunasa sauti - Hivi ni vifaa vinavyotumiwa kurekodi sauti za watu wanapozungumza ama kutamka. Mtalaa huu unapendekeza kwamba mwalimu arekodi sauti ya mweledi wa matamshi akitamka fonimu za Kiswahili kisha akawachezea wanafunzi rekodi hiyo wakisikiliza na kutamka yale wanayosikia yakitamkwa.

(b) Rununu - Rununu ni simu ya mkononi. Mwalimu anaweza kutumia simu kurekodi sauti za mtu akitamka fonimu za Kiswahili kisha akatumia rekodi hiyo kuwafunza wanafunzi. Vilevile, kwa sababu simu ina uwezo wa kupiga picha, mwalimu anaweza kupiga picha za vitu mbalimbali ambavyo wanafunzi watajifunza kutamka majina yavyo.

(c) Vitu halisi - Hivi ni vitu ambavyo hupatikana katika mazingira ya kawaida. Mwalimu hutumia vitu halisi kama vile matunda, vikombe na kalamu kuwafundisha wanafunzi jinsi ya kutamka majina hayo na katika harakati hizo, wanajifunza fonimu za Kiswahili. Vitu halisi huwachangamsha wanafunzi na kuwatia motisha ya kujifunza.

(d) Vipakatalishi - Hizi ni tarakalishi ndogo ambazo huwa rahisi kubeba na kutumiwa mahali popote. Vipakatalishi vina sifa na uwezo mbalimbali ambazo humwezesha mwanafunzi na mwalimu kufanikisha ufundishaji na ujifunzaji wa fonimu za Kiswahili. Kwanza, vipakatalishi vina uwezo wa kuhifadhi video kama vile za vibonzo vikitamka fonimu za Kiswahili na hivyo kuwachangamsha wanafunzi wanapojifunza fonimu za Kiswahili. Vipakatalishi vilevile huweza kuhifadhi picha na mazoezi mbalimbali yanayofanywa na wanafunzi hivyo kuboresha ufundishaji na ujifunzaji wa fonimu za Kiswahili.
Mbinu nyingine za ufundishaji zinazopendekezwa na mtalaa wa umilisi wa 2.6.6.3 ni kama vile televisheni, tabuleti na redio.

\section{Ufaafu wa Mbinu za Ufundishaji wa Fonimu za Kiswahili katika Kiwango cha Chekechea katika Mtalaa wa 8.4.4 na wa Umilisi wa 2.6.6.3}

Mbinu zilizopendekezwa kutumiwa katika ufundishaji wa fonimu za Kiswahili katika kiwango cha chekechea kwa mujibu wa mtalaa wa 8.4.4 na wa 2.6.6.3 zina ufaafu ufuatao:

(a) Chati - Mbinu hii iliwafaa wanafunzi kwa vile ilimpa mwalimu uwezo wa kutathmini matamshi ya wanafunzi wanapokuwa katika vikundi vidogo vidogo na kuwarekebisha wale wasioweza kutamka kwa usahihi.Vilevile, chati moja ingetumiwa kuwasomesha wanafunzi wengi kwa sababu inapoangikwa ukutani, hata darasa zima lingeweza kuiona na kusoma fonimu za Kiswahili kwa urahisi.

(b) Picha - Picha ilikuwa bora katika ufundishaji wa fonimu za Kiswahili kwa sababu ilikuwa rahisi kwa wanafunzi kutaja majina ya wanyama na vitu ambavyo wanaviona kwenye picha kuliko vile ambavyo hawavioni, na tena kuwapa uelewa bora ya mazingira yao. Picha pia ina sifa ya kuteka makini ya wanafunzi na kuwapa ari ya kusalia ndani ya somo kwa muda bila ukinaifu.

(c) Kadi - Kadi zilikuwa muhimu kwa sababu zingemwezesha mwalimu kujua idadi ya fonimu ambazo wanafunzi wanapata taabu kuzitamka kisha akazitenga na kusisitiza baadaye. Kadi pia ziliwafaa wanafunzi kwa sababu ilikuwa rahisi kwao kusoma fonimu pweke zinazoandikwa kwenye kila kadi.

(d) Kanda za kunasa sauti - Sauti inaporekodiwa, inaweza kuhifadhiwa na kutumiwa kufundishia kwa muda mrefu kuliko sauti zinazotamkwa moja kwa moja.

(e) Rununu - Ni rahisi kutumia rununu darasani na nyumbani hivyo kurahisisha ujifunzaji. Pia, rununu humpa mwanafunzi ujuzi wa kidijitali ambao ni muhimu katika ulimwengu wa leo.

7 | This work is licensed under a Creative Commons Attribution 4.0 International License. 
(f) Vitu halisi - Vitu halisi huwapa wanafunzi motisha ya kuhusika katika ujifunzaji anapoviona na kutamka majina.

(g) Vipakatalishi - Humpa mwanafunzi ujuzi wa kidijitali.

\section{Udhaifu wa Mbinu za Ufundishaji wa Fonimu za Kiswahili katika Kiwango cha Chekechea katika Mtalaa wa 8.4.4 na wa Umilisi wa 2.6.6.3}

Mbinu zilizopendekezwa kutumiwa katika ufundishaji wa fonimu za Kiswahili katika kiwango cha chekechea kwa mujibu wa mtalaa wa 8.4.4 na wa 2.6.6.3 zina udhaifu ufuatao:

(a) Chati - Mwalimu asipokuwa makini, hawezi kutambua mwanafunzi anayepata matatizo katika kutamka baadhi ya fonimu kwa sababu inakuwa rahisi kwa mwanafunzi kama huyo kujificha ndani ya wenzake, kwa sababu chati aghalabu husomwa pamoja na darasa zima.

(b) Picha - Wanafunzi wenye mahitaji maalum kama vile wasio na uwezo wa kuona vizuri wanaweza kupata matatizo kuziona picha na michoro hivyo kupitwa na hoja muhimu kuhusu fonimu za Kiswahili.

(c) Kadi - Kwa sababu kadi aghalabu huandikwa fonimu moja moja, mwanafunzi anaweza kupata taabu kutambua mfuatano wa fonimu za Kiswahili katika msururu wa abjadi.

(d) Kanda za kunasa sauti - Kanda hizi aghalabu ni ghali na ni shule chache tu zilizo na uwezo wa kuzinunua na kuzitumia kufundishia darasani.

(e) Rununu - Wanafunzi wakitumia rununu bila kudhibitiwa vyema, wanaweza kufungua mitandao yenye mambo yanayokiuka maadili ya jamii.

(f) Vitu halisi - Kuna vitu halisi ambavyo huenda vikahitajika darasani lakini vikawa vigumu kubeba na kutumiwa. Kwa mfano, ng'ombe, simba na bahari.

(g) Vipakatalishi - Kando na vipakatalishi kuhitaji ujuzi mkubwa wa kidijitali, haviwezi kutumiwa mahali pasipo na nguvu za umeme wala pasipo na usalama wa kuhakikisha kwamba haviibiwi na kuharibiwa.

\section{MATOKEO KIULINGANISHI}

Makala haya yamedhihirisha kuwa, mbinu zilizopendekezwa na mtalaa wa umilisi wa 2.6.6.3 zina vipengele vingi vya ubora ikilinganishwa na zile zilizopendekezwa na mtalaa wa 8.4.4 katika ufundishaji wa fonimu za Kiswahili. Ubora wa mbinu zilizopendekezwa na mtalaa wa umilisi ni kwamba, zinazingatia ukuaji wa mwanafunzi darasani na nje ya darasa. Ni mbinu zinazompa mwanafunzi nafasi ya kujenga umilisi wake wa fonimu za Kiswahili sambamba na kukuza vipaji vyake mbalimbali teknolojia ikiwemo. Mbinu zilizopendekezwa na mtalaa wa 8.4.4, kwa upande mwingine, zilikuwa na msisitizo wa umilisi wa maarifa ya darasani bila kuzingatia ukuaji wa mwanafunzi nje ya darasa.

Kwa kuzingatia tofauti hizi, inakuwa wazi kwamba, matokeo ya mbinu za ufundishaji za mtalaa wa umilisi wa 2.6.6.3 ni bora kuliko yale ya mbinu zilizopendekezwa na mtalaa wa 8.4.4.

\section{MAPENDEKEZO}

Makala haya yameonyesha kuwa mbinu za ufundishaji wa fonimu za Kiswahili zilizopendekezwa na mtalaa wa umilisi wa 2.6.6.3 zina uwezo wa kutoa matokeo bora zaidi jinsi ilivyoangaziwa awali. Ili kuimarisha matokeo haya katika ufundishaji wa fonimu za Kiswahili katika viwango vya chekechea, makala haya yanapendekeza yafuatayo:

I. Wizara ya elimu kwa kushirikiana na serikali za kaunti inafaa kuimarisha miundomsingi katika viwango vya chekechea ili kuwezesha matumizi ya vifaa vya kiteknolojia kama vile, vipakatalishi na kanda za video. Kama ilivyotokea, vifaa hivi vinahitaji nguvu za umeme na usalama ili vitumike ifaavyo.

II. Walimu na wazazi wanapaswa kupewa mafunzo zaidi ya jinsi ya kutumia vifaa vya kiteknolojia wanapowaongoza wanafunzi kuvitumia. Waweze kuwaelekeza wanafunzi kufungua mitandao salama ili wasiathirike na mambo yanayokiuka maadili ya kijamii

8 This work is licensed under a Creative Commons Attribution 4.0 International License. 
ambayo wakati mwingine hutundikwa mtandaoni kinyume na sheria.

III. Kwa sababu nyingi za mbinu zilipondekezwa na mtalaa wa 2.6.6.3 kutumiwa katika ufundishaji wa fonimu za Kiswahili zinahitaji muda mwingi ili zitumike kwa ukamilifu darasani, wizara ya elimu inafaa kuongeza muda wa ufundishaji hasa wakati mwalimu na wanafunzi wanapotumia vifaa hivyo darasani.

IV. Wizara ya elimu ya kitaifa kwa ushirikiano na wizara za elimu katika kaunti mbalimbali zinafaa kufanya ukaguzi na uchunguzi wa kila mara ili kuhakikisha kwamba walimu wanaowafundisha wanafunzi wanawafundisha kwa namna inayofaa na ya kwamba matokeo yanayotarajiwa yanaafikiwa.

\section{HITIMISHO}

Makala haya yamefafanua kiulinganishi mbinu mbalimbali za ufundishaji wa fonimu za Kiswahili katika viwango vya chekechea kwa mujibu wa mtalaa wa 8.4.4 na wa umilisi 2.6.6.3. Imeeleza maana ya mtalaa pamoja na kufafanua sifa za mtalaa bora. Vilevile, maana ya fonimu kwa jumla imetolewa kwa mujibu wa wataalamu mbalimbali huku aina za fonimu ambazo ni irabu na konsoanti zikitolewa maelezo toshelevu. Mfanano na tofauti za mbinu za ufundishaji kwa mujibu wa mtalaa wa 8.4.4 na wa umilisi wa 2.6.6.3 zimeelezwa. Vilevile, makala haya yamefafanua kuhusu ufaafu na udhaifu wa mbinu hizo za ufundishaji wa fonimu za Kiswahili. Mwisho, matokeo kiulinganishi yameelezwa pamoja na kuonyesha msimamo wa makala haya yana mapendekezo yake.

\section{MAREJELEO}

Bruner, J. S. (1974). The Relevance of Education. New York: Norton.

Chiduo, F. K. na wengine. (2016). Kamusi Elezi ya Kiswahili. Nairobi: Jomo Kenyatta Foundation.

Choge, S. (2009). Understanding Kiswahili Vowels. Journal of Pan African Studies, 2(8), 62-77.

Facun-Granadozo, R. (2014). Developing Mastery in Phonemic Awareness, Phonics, and 9 | This work is licensed under a Creative Commons Attribution 4.0 International License.
Morphemic Awareness: A Multiple Case Study of Preservice Early Childhood Educators. PhD Thesis. East Tennessee State University.

Flinders, D. \& Thornton, J. S. (2004). The Curriculum Studies Reader. Milton Park, Abingdon, Oxon (UK): Routledge Falmer.

KICD. (2017). Curriculum Design for PP1, PP2 \& Grades One to Three. Nairobi: Kenya Institute of Curriculum Development.

KICD. (2019). Curriculum Design for Grade Four. Nairobi: Kenya Institute of Curriculum Development.

Kridel, C. (Ed.) (2010). Encyclopaedia of Curriculum Studies. A Thousand Oaks (CA): Sage Publishers.

Massamba, D. P. B., Kihore, Y. M, \& Msanjila, Y. P. (2004).Fonolojia ya Kiswahili Sanifu. Dar es salaam: TUKI.

Mdee, J. S. na wengine (2019). Kamusi ya Karne ya 21. Nairobi: Longhorn Publishers PLC.

Mgullu, S. R. (2016). Mtalaa wa Isimu: Fonetiki, Fonolojia na Mofolojia ya Kiswahili. Nairobi: Longhorn Publishers PLC.

Mwongozo wa KIE .(2002). Primary School Syllabus. Nairobi: Kenya Institute of Education

Okal, O. B. (2017). Uhakiki wa Fonimu na Miundo ya Silabi za Kiswahili: Kioo cha Lugha Juz 13.

Piaget, J. (1972). "Development and Learning." In Lavatellic S. \& Stendler F. (wah) (1972) Reading in Child Behaviour and Development. New York, Hacourt Brace.

Powers, E. J. (2008). 'The Effectiveness of Phonemic Awareness Instruction on Struggling Readers'. Masters Thesis, State University of New York College at Brockport.

Wandera, S. (2018). Mitalaa ya Shahada ya Uzamili ya Kiswahili: Mtazamo wa Urie Bronfenbrenner. Coretrain Journal of Languages, Humanities, Social Sciences and Education, 1(1), 236-248. 